\title{
An anti-DEC-205 monoclonal antibody stimulates binding of thymocytes to rat thymic dendritic cells and promotes apoptosis of thymocytes
}

\author{
IVANA MAJSTOROVIĆ ${ }^{\prime}$ DRAGANA VUČEVIĆ ${ }^{\prime, 2}$, BOJAN PAVLOVIĆ \\ MIODRAG ČOLIĆ,3 \\ Institute of Medical Research, Military Medical Academy, Belgrade, Serbia \\ ${ }^{2}$ Medical Faculty, Military Medical Academy, University of Defence, Belgrade, Serbia \\ ${ }^{3}$ Medical Faculty, University of Niš, Niš, Serbia
}

\begin{abstract}
DEC-205, a transmembrane receptor responsible for cross-presentation of apoptotic cell-derived antigens, is expressed by cortical thymic epithelial cells (TEC) and thymic dendritic cells (TDC) in humans and mice, but its function in T-cell development is still unclear. In this work we have studied for the first time the expression of DEC-205 in the rat thymus by HD83 monoclonal antibody $(\mathrm{mAb})$ and immunohistochemistry, as well as the ability of this mAb to modulate thymocyte - TDC interactions in vitro. We showed the positivity of cortical TEC in situ, including thymic nurse cells (TNC) in suspension, and TDC, whereas subcapsular, perivascular and medullary TEC were negative. All examined DEC-205 positive and DEC-205 negative structures were MHC class II positive. HD83 mAb increased apoptosis of thymocytes in co-culture with TDC in vitro and the process was associated with increased binding of thymocytes to TDC in a rosette form. Since negative selection of thymocytes by clonal deletion (apoptosis) was mediated predominantly by TDC, our results suggest the possible indirect effect of the DEC-205 molecule in these mechanisms.
\end{abstract}

Key words: rat thymus, TDC, DEC-205, thymocyte apoptosis.

(Centr Eur J Immunol 2014; 39 (4): 411-418)

\section{Introduction}

T-cell differentiation and repertoire selection in the thymus are essential for the establishment and maintenance of the adaptive immune system, and play a critical role in immunological tolerance [1].

The mechanisms of positive and negative selection are central for the generation of self-tolerant T-cells, capable of recognizing foreign antigens in the context of self-MHC molecules [2]. These processes require various inputs from surrounding thymic stromal cells composed of thymic epithelial cells (TEC), thymic dendritic cells (TDC), macrophages and other cells (fibroblasts, endothelial cells, etc.) which provide an array of optimal signals to developing thymocytes. Substantial evidence now supports the notion that major inducers of positive selection of thymocytes are cortical TEC, whereas TDC and medullary TEC contribute to the negative selection [3]. Many of the signals required for different stages of intrathymic development are provided by thymic stromal cells in the form of cell surface molecules [1]. Although intrathymic
T-cell differentiation and selection have been an area of intense investigation, the molecular and cellular mechanisms of these processes are not resolved yet.

Previous investigations have shown that DEC-205 (CD205), a $205 \mathrm{kDa}$ integral membrane glycoprotein, positively discriminates cortical TEC $[4,5]$. Expression of DEC-205 was detected also on antigen presenting cells, especially on DC in many different tissues including TDC $[4,6]$. Studies investigating the CD205 expression by peripheral DC have demonstrated that CD205 operates as an antigen uptake and processing receptor [5]. Human and mouse DEC-205 antigen exhibits approximately $80 \%$ protein homology, suggesting its conserved function between species [7]. Park et al. [8] successfully generated anti-human DEC-205 monoclonal antibodies (mAbs), produced from human DEC-205-immunized DEC-205 knock-out mice, which were species cross-reactive. One of these mAbs, named HD83, was found to recognize DEC-205 antigen in rat spleen and lymph node [8]. To date, there has

Correspondence: Prof. Miodrag Čolić, Medical Faculty, Military Medical Academy, University of Defence, Crnotravska 17, 1100 Belgrade, Serbia, e-mail: fakultet.vma@mod.gov.rs 
been no report on the expression status and the potential functional significance of DEC-205 in the rat thymus.

In an attempt to delineate the function of rat DEC-205 in thymocyte development we characterized its expression within the rat thymus by using HD83 mAb and an immunohistochemical approach. We also analyzed the adhesive interactions between thymocytes and TDC in vitro and showed that HD83 mAb enhanced the binding of thymocytes to TDC and stimulated thymocyte apoptosis.

These results suggest the possible molecular mechanisms through which DEC-205 antigen may influence T-cell development and selection in the thymus.

\section{Material and methods}

\section{Animals}

Autoshaping-operant (AO) rats, male, 6-8 weeks old, bred at the Farm for Experimental Animals, Military Medical Academy (MMA) (Belgrade, Serbia) were used in this study. The study was approved by the Ethical Committee of MMA, Belgrade, Serbia, according to the Guidelines for Animal Study, № 282-12/2002.

\section{Antibodies}

The following mouse anti-rat monoclonal antibodies (mAbs) were used: HD83, mouse anti-DEC-205 mAb cross-reactive with human, mouse, monkey and rat DEC205 antigen, a kind gift from Dr. Chae Gyu Park (Severance Biomedical Science Institute, Yonsei University College of Medicine, Korea) [8] and OX-6 mAb, (anti I-A) (Serotec). As an irrelevant $\mathrm{mAb}$, the isotype matching $\mathrm{mAb}$ VAM 4.1, reactive with Pseudorabies virus and nonreactive with rat antigens, produced at the Institute for Medical Research, MMA, Belgrade, was used. Horseradish peroxidase (HRP)-conjugated rabbit anti-mouse immunoglobulin (Ig) was purchased from Dako, Denmark.

\section{Immunohistochemistry}

Cryostat sections $(6-8 \mu \mathrm{m})$ of rat thymus were fixed in acetone for 10 minutes at $-20^{\circ} \mathrm{C}$, air dried and rehydrated in $50 \mathrm{mM}$ Tris-buffered saline (TBS) pH 7.6. Slides were then incubated overnight at $4{ }^{\circ} \mathrm{C}$ with appropriate dilutions of mAbs (final concentrations $10 \mu \mathrm{g} / \mathrm{ml}$ for all tested $\mathrm{mAbs}$ ) and washed in TBS. After that, incubation with HRP-conjugated rabbit anti-mouse Ig (1:25) in TBS containing 5\% normal rat serum for 30 minutes followed. After washing in TBS the specific immunolabeling was visualized by adding $0.06 \%$ diaminobenzidine (DAB) (Serva) and $0.01 \%$ hydrogen peroxide (Sigma) in TBS. Finally, the sections were washed and then lightly counterstained with haematoxylin, prior to mounting. Micrographs of stained thymus sections were taken under the conventional light microscope (Olympus, BX 41). The specificity of immunostaining was confirmed by omission of primary mAb.

\section{Immunocytochemistry}

Cytospins of TDC rosettes were fixed in pararosaniline for 2 minutes, washed in TBS and incubated with appropriate dilution of $\mathrm{HD} 83 \mathrm{mAb}(10 \mu \mathrm{g} / \mathrm{ml})$ for 60 minutes. After washing in TBS, slides were incubated with HRP-conjugated rabbit anti-mouse Ig (1:25) with addition of 5\% normal rat serum. Specific immunolabeling was visualized by adding $0.06 \%$ diaminobenzidine (DAB) (Serva) and $0.01 \%$ hydrogen peroxide (Sigma) as already described. The slides were lightly counterstained with haematoxylin, prior to mounting. The control was VAM 4.1, used as an irrelevant $\mathrm{mAb}$ at the same concentration.

\section{Thymic dendritic cells preparation and cultivation}

Thymic dendritic cells were isolated from rat thymuses by using the method previously described by Vasilijic et al. [9]. Briefly, $4 \mathrm{ml}$ of thymocyte cell suspension (3-4 $\times 10^{8}$ cells) was overlaid on $3 \mathrm{ml}$ of OptiPrep ${ }^{\mathrm{TM}}$ gradient (Nycomed Pharma AS, Oslo, Norway) (density $1.078 \mathrm{~g} / \mathrm{ml}$ and osmolarity $390 \mathrm{mOsm}$ ). Cells were then centrifuged at $600 \mathrm{~g}$ for $15 \mathrm{~min}$ at $4^{\circ} \mathrm{C}$ and TDC fraction was collected at the RPMI medium/Optiprep interface. The purity of such prepared TDC was $40 \%$, as checked by morphology. Contaminating cells were mostly thymocytes and macrophages. To further enrich the TDC suspension, TDC were cultivated overnight in 6-well plates (Flow, ICN) at a concentration of $1.5 \times 10^{6}$ cells $/ \mathrm{ml}$ of RPMI 1640 medium supplemented with $10 \%$ fetal calf serum (FCS) and $5 \mathrm{ng} / \mathrm{ml}$ mouse recombinant granulocyte macrophage-colony stimulating factor (rGM-CSF) (Genzime, Boston, USA) at $37^{\circ} \mathrm{C}$ and $5 \% \mathrm{CO}_{2}$. Thymic dendritic cells were collected as non-adherent cells, resuspended in warm RPMI 1640 medium with $0.02 \%$ Na-EDTA and purified again over OptiPrep ${ }^{\mathrm{TM}}$ density gradient (Sigma). After washing twice in RPMI 1640 with $2 \%$ FCS, the TDC suspension was counted and analyzed in Turk's solution under the microscope. The purity of cultivated TDC was around $80 \%$.

\section{Thymocytes}

To prepare thymocytes, the thymus was teased with a syringe plunge, cells were collected, filtered through a nylon mesh, centrifuged for 10 minutes at $600 \times \mathrm{g}$, and washed in 1640 RPMI medium with $2 \%$ FCS. The thymocyte suspension was counted, and cell viability, which was higher than $95 \%$, was checked by Trypan blue exclusion.

\section{Rosette assay}

Thymic dendritic cells $\left(5 \times 10^{4}\right)$ were preincubated with HD83 mAb, control VAM $4.1 \mathrm{mAb}$ (both at the concentrations of $10 \mu \mathrm{g} / \mathrm{ml}$ ), or medium alone for $15 \mathrm{~min}$, then mixed with freshly isolated thymocytes $\left(2.5 \times 10^{5}\right), 1: 5$ ratio, in $20 \mu \mathrm{l}$ of the complete medium, transferred into a Terasaki microwell plate and cultured in a hanging drop. After 30 minutes 
of incubation at $37^{\circ} \mathrm{C}$ and $5 \% \mathrm{CO}_{2}$, the cells were gently collected and cytocentrifuged onto glass slides. Cytospins were stained with May-Grunwald Giemsa (MGG) and analyzed by light microscopy. Thymic dendritic cells that bound three or more thymocytes were scored as rosettes. For each assay 200-500 TDC were counted and each determination was performed in duplicate. Two parameters were determined: rosette formation (represented by the percentage of TDC forming rosettes in comparison with a total number of TDC) and mean number of thymocytes per rosette.

\section{Apoptosis assays}

Purified cultivated TDC were mixed with syngeneic thymocytes at $1: 5$ ratio and cultivated in RPMI 1640 medium with $10 \%$ FCS in 96-well flat bottom plates. HD83 mAb or control VAM $4.1 \mathrm{mAb}$ (both $10 \mu \mathrm{g} / \mathrm{ml}$ ) were added to TDC/thymocyte co-cultures. After $6 \mathrm{~h}$ incubation thymocyte apoptosis was measured. The percentage of apoptotic thymocytes, identified in TDC/thymocyte co-cultures, was determined by morphological criteria as previously described [10] and AnnexinV-FITC/Propidium Iodide (PI) method. For morphological evaluation, thymocytes cultivated with TDC were washed with PBS, fixed with Turck's solution and analyzed by light microscopy. In each sample at least 500 cells were counted. Apoptotic cells were identified by condensation of chromatin, nuclear pyknosis and/or karyorrhexis. The results were expressed as a percentage of apoptotic thymocytes relative to total counted thymocytes. For cytometric determination of thymocyte apoptosis, cells were stained with AnnexinV-FITC/PI kit KXN50 (R\&D, Abingdon, Oxon, UK). Cells from each well were stained according to the manufacturer's instructions and analyzed using a flow cytometer (CyFlow Cube 6, Partec, Germany). Double negative cells are viable cells, AnnexinV-FITC ${ }^{+} / \mathrm{PI}^{-}$cells represent thymocytes in the early stage of apoptosis, double positive cells are late apoptotic/secondary necrotic thymocytes, whereas only $\mathrm{PI}^{+}$cells are primarily necrotic cells.

Apoptosis of thymocytes within TDC rosettes on cytospins prepared after 30 minutes of incubation, was determined also based on morphological criteria. The results were expressed as \% of apoptotic thymocytes within rosettes relative to total thymocytes bound to TDC.

\section{Statistical analysis}

Results were expressed as mean \pm SD. Statistical analysis was performed by $t$-test. $P<0.05$ was considered statistically significant.

\section{Results}

\section{Expression of DEC-205 antigen in the rat thymus}

The sections of rat thymuses were stained with HD83 $\mathrm{mAb}$. Results presented in Figure 1 clearly show that this an- tibody stains cortical epithelium and isolated stellate or ramified cells in the medulla, resembling TDC. The staining of the cortex was stronger than in the medulla, especially on the border of epithelial-free zones, which were clearly negative, and at the cortico-medullary zones. Subcapsular epithelial cells, perivascular epithelial cells in the cortex and medullary epithelial cells were DEC-205 negative. By comparing the staining of the thymus with OX-6 mAb (an anti-rat MHC class II molecule antibody), it can be seen that both cortical and medullary epithelial cells, including subcapsular/perivascular TEC, TDC in the medulla and macrophage-like cells throughout the whole section, including macrophages within epithelial-free zones, express MHC class II molecules.

The positive immunoreaction of isolated thymic nurse cells (TNC) and TDC (Fig. 2), and negative staining of both viable and apoptotic thymocytes by HD 83 mAb (data not shown), confirmed the pattern expression of the DEC205 antigen seen in situ.

\section{HD83 mAb stimulates the formation of rosettes between thymocytes and thymic dendritic cells}

To study possible functional roles of the DEC-205 antigen in the thymus, we performed a rosette assay between thymocytes and TDC in vitro. Figure 3 shows that HD $83 \mathrm{mAb}$ stimulates the rosette formation, compared to corresponding controls, as judged by either the percentage of TDC forming rosettes or by the mean number of thymocytes per rosette.

\section{HD83 mAb stimulates apoptosis of thymocytes}

Thymocytes die rapidly in culture and the apoptotic process was accelerated in co-culture with TDC. Both morphological and flow-cytometric methods gave similar results (Fig. 4A and 4B). When HD83 mAb was added to the co-culture, apoptosis of thymocytes was further increased, compared to controls. The process was more evident when early stage apoptotic cells (AnnexinV-FITC ${ }^{+}$/ $\mathrm{PI}^{-}$) were analyzed (Fig. 4B). To check whether this process was associated with increased adhesiveness of thymocytes to TDC, apoptosis was measured separately in rosettes. It can be seen that the percentage of apoptotic thymocytes in rosettes was higher compared to apoptosis of total thymocytes. The percentage of apoptotic thymocytes in rosettes (stimulated in the presence of HD83 mAb) was statistically significantly higher compared to the percentage of apoptotic thymocytes in rosettes in control cultures (without HD83 mAb or in the presence of Ir mAb). This finding suggests that the increased formation of TDC/thymocyte rosettes was associated with increased apoptosis of thymocytes within these cell-to-cell clusters.

\section{Discussion}

The first mAb produced against mouse DEC-205, NLDC-145, has been shown to stain cortical TEC and 

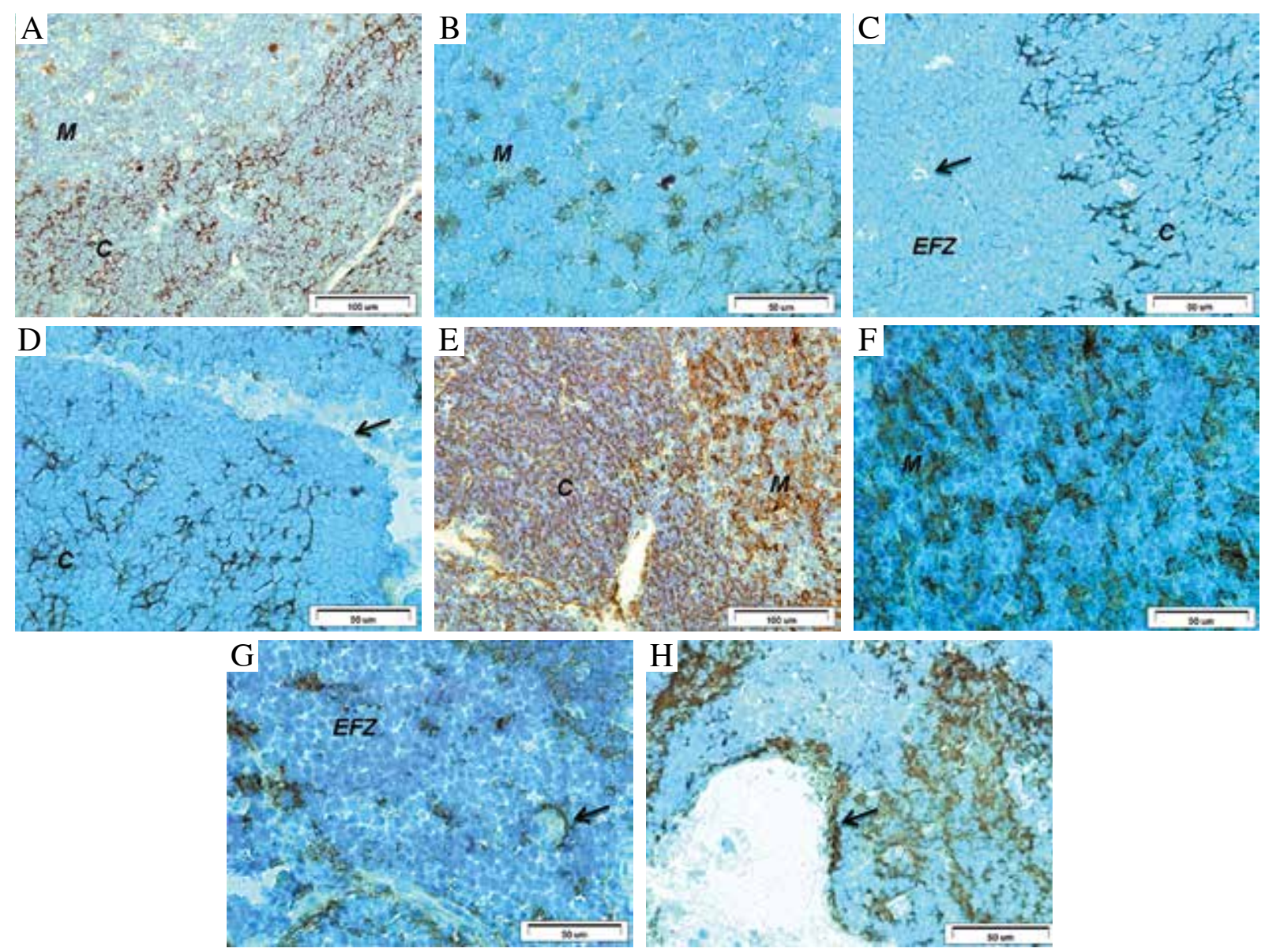

$M$ - medulla; $C$ - cortex; $E F Z$ - epithelial-free zone

Fig. 1. Immunoperoxidase staining of rat thymus, with HD83 mAb (A-D) and anti-MHC II mAb (E-H). Specific HD83 labeling is localized in the cortex $(\mathbf{A})$ and less intense on some individual dendritic-like cells scattered in the medulla (B). Note stronger HD83 labeling of cortical epithelium, specifically at the cortico-medullary (A) and cortico-epithelial-free zones border (C). Perivascular epithelial cells (arrow) (C) and subcapsular epithelial cells (arrow) (D) are HD83 negative. Staining with OX-6 mAb shows the positivity of both cortical and medullary compartment (E). Note strong staining of medullary TEC and TDC (F), isolated macrophages and perivascular TEC (arrow) in epithelial-free zones (G), subcapsular epithelium (arrow) and cortical epithelium (H). Magnifications: A and E 20×; others 40×

medullary TDC [11]. Subsequent studies confirmed the expression of DEC-205 on mouse DC in other lymphoid organs, especially on the CD8+ DC subset and weakly on other antigen-presenting cells (macrophages and B-cells) $[4,12-14]$. Later on, the study in the human system showed distribution of DEC-205 on other cells, including myeloid blood DC and monocytes, but also at low levels on natural killer cells, plasmacytoid DC and T-cells [6]. Since the data related to DEC-205 expression in the rat system are very scarce [8], the first aim of this study was to characterize the expression of this molecule in the rat thymus. By using an immunohistochemical approach on rat thymus sections and isolated cells, we confirmed that the pattern expression of DEC-205 closely resembled that in the mouse thymus. Namely, cortical TEC, including TNC, and medullary TDC were strongly DEC-205 positive. It is interesting that subcapsular and perivascular epithelial cells in the cortex were DEC-205 negative. In contrast, all thymic epithelial structures, TDC and macrophages were MHC class II positive. These findings, together with negative staining of medullary TEC with HD83 mAb are in accordance with already published phenotypic heterogeneity of TEC [15]. An extensive immunohistochemical study demonstrated that subcapsular/perivascular and most medullary TEC share the same phenotype, in contrast to the cortical TEC, which express some specific markers [15]. It is obvious that such a unique cortical TEC-subset-specific marker is DEC-205. 
A
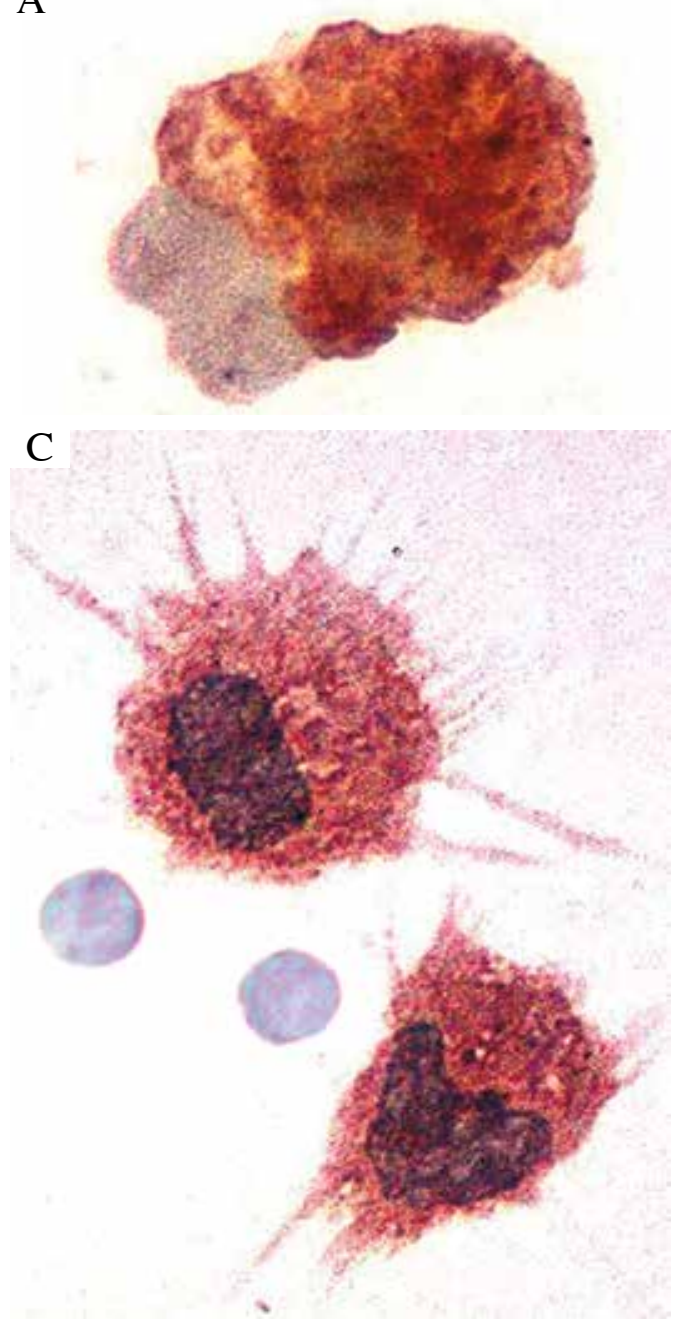

It is known that DEC-205 belongs to the macrophage mannose receptor family of C-type lectin endocytic receptors, which behaves as an antigen-uptake/processing receptor for DC and cortical TEC [5]. Its predominant function is to direct capture of antigens from the extracellular space to a specialized antigen-processing compartment [16]. Based on these results it can be postulated that DEC205 must have a role in endocytosis and subsequent processing of self-antigens in the thymus. Such mechanisms are important for selection processes in the thymus [17]. A paper published by Small and Kraal [18] showed an in vitro evidence for participation of DEC-205 in clearance of apoptotic thymocytes by mouse cortical TEC. Later on, such a role has been postulated for mouse CD8+ DC subset, which selectively enodocytoses dying cells in culture and in vivo [14]. This finding is very important because CD8+ DC, in contrast to CD8- DC, are specialized to capture dying cells and to cross-present cellular antigens to both CD4+ and CD8+ T-cells, where DEC-205 might
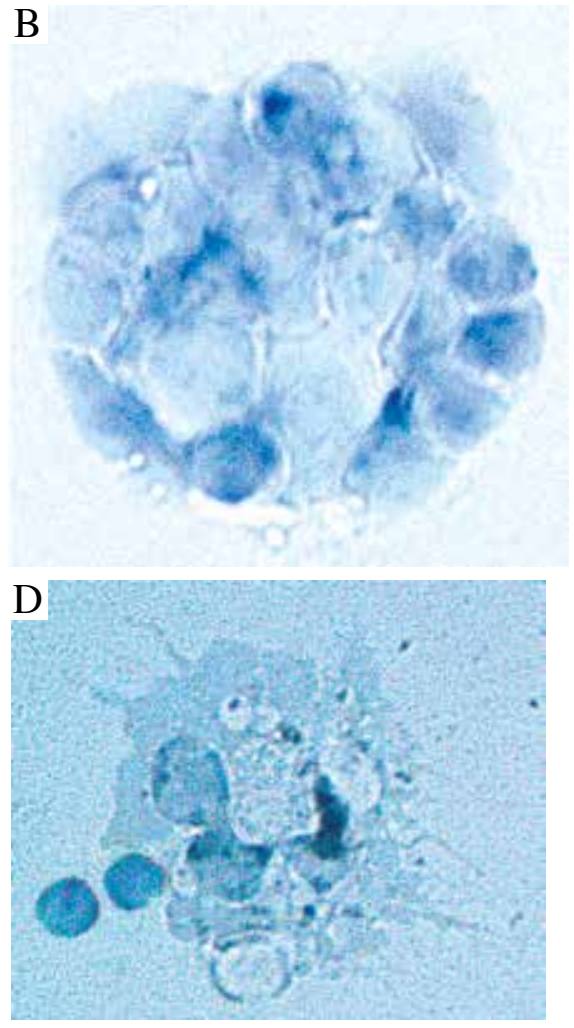

Fig. 2. Expression of DEC-205 on thymic stromal cells in vitro. TDC/thymocyte rosettes, established in vitro after 30 min of cell incubation, as described in Material and methods, were labeled with the immunoperoxidase staining. Note the positivity of TNC (A) and TDC (C) compared to Ir mAb controls (B and $\mathbf{D}$, respectively) (magnifications 40x). Thymocytes were negative (C)

have a very important role. The ligands for DEC-205 in the tissue have not been identified yet. The only paper that deals with this research area showed evidence that $\mathrm{CpG}$ oligonucleotides, well known pathogen associated molecular pattern and a selective TLR-9 agonist, bind to both mouse and human DEC-205 [19]. It has been recently confirmed that apoptotic thymocytes express ligands for mouse DEC-205, as demonstrated by using a DEC-205 Ig fusion protein [20].

All these results motivated us to study the possible role of rat DEC-205 in the processes of thymocyte apoptosis. Our previous results showed that rat TDC in culture stimulated apoptosis of thymocytes. This process was associated with binding of thymocytes to TDC and was partially blocked by a LFA-1 mAb [21]. Such results were confirmed later by other authors [22] by demonstrating the involvement of TDC- and thymic macrophages-derived $\mathrm{NO}$, as an important effector mechanism in apoptosis induction of rat thymocytes. To see whether DEC-205 inter- 

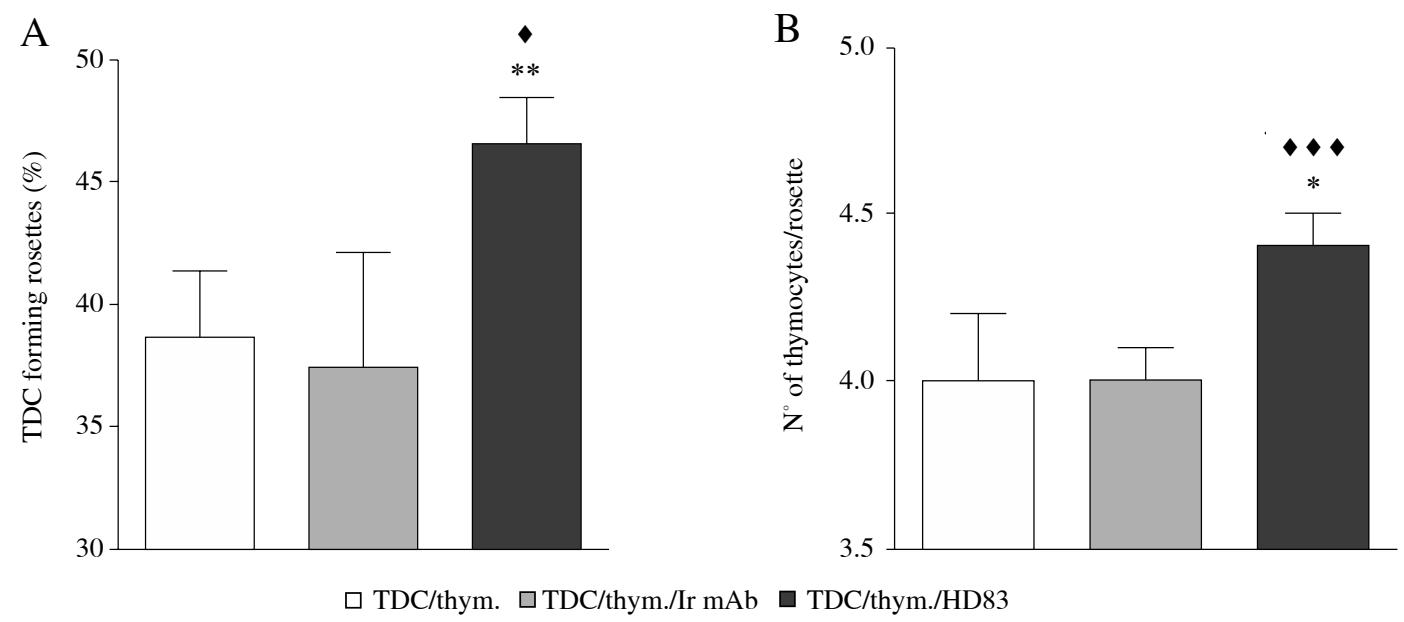

$\mathrm{C}$ C Ir mAb
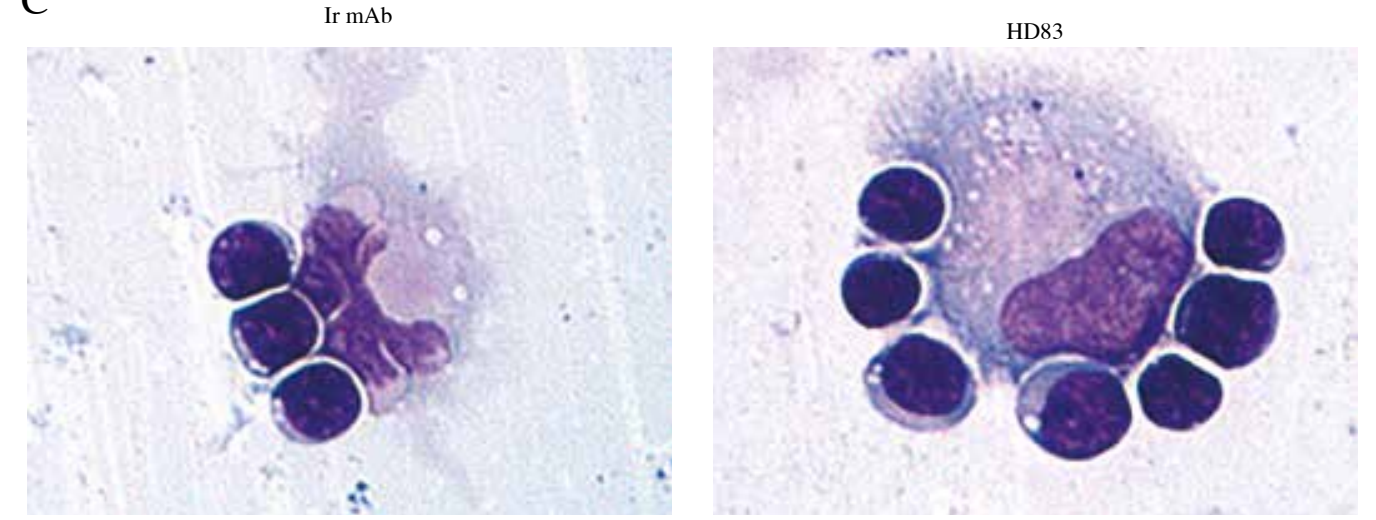

${ }^{*} p<0.05 ;{ }^{* * *} p<0.01$ compared to Ir mAb, ${ }^{*} p<0.05 ;{ }^{\cdots *} p<0.001$ compared to TDC/thymocytes

Fig. 3. Effect of HD83 mAb on formation of TDC/thymocyte rosettes. The percentage of TDC forming rosettes after 30 min culture (A). The mean number of thymocytes per rosette (B). Microscopic analysis of TDC/thymocyte rosettes after 30 min incubation with TDC in the presence of HD83 or irrelevant mAb (C) (magnification: 40×), MGG staining. The results were given as mean \pm SD (3 different experiments) after 200-500 cells with or without rosettes (A) or thymocytes in rosettes $(\mathbf{B})$, were calculated in each experiment

feres with binding of thymocytes to TDC, we performed a rosette assay and demonstrated that HD83 mAb significantly increased these cell-to-cell adhesive interactions. The process was associated with increased apoptosis of thymocytes in co-culture with TDC and with an increased percentage of apoptotic cells in rosettes.

These findings suggest an active role of TDC in induction of thymocyte apoptosis, thus mimicking the mechanisms of negative selection [17]. However, an increase in the percentage of apoptotic thymocytes in TDC/thymocyte rosettes may also suggest a role of TDC in preferential binding of apoptotic thymocytes. If this hypothesis that DEC-205 on TDC binds to apoptotic thymocytes is true, based on previously published results on cortical TEC- or CD8+ DC-thymocyte interactions [14, 18], one can expect that anti-DEC-205 mAb inhibits these processes. Our op- posite results may suggest that HD83 might increase adhesion of thymocytes to TDC, including apoptotic ones, non-specifically, by modulating binding domains on DEC205. It is known that mouse and human DEC-205 molecules, showing about $80 \%$ homology, are C-type multilectin receptors with a large extracellular portion containing an $\mathrm{N}$-terminal cysteine rich domain, a fibronectin type II domain and 10 domains structurally (although not necessarily functionally) resembling C-type lectin-like domains $[5,7]$ also known as carbohydrate recognition domains (CRDs). At the moment it is not known whether similar domains are present in rat DEC-205 and which molecules or molecular residues on rat thymocytes, especially on apoptotic cells, bind to these domains. It has been shown only that HD83 mAb binds to an epitope on CRD1 and CRD2 on DEC-205 molecule [8]. 

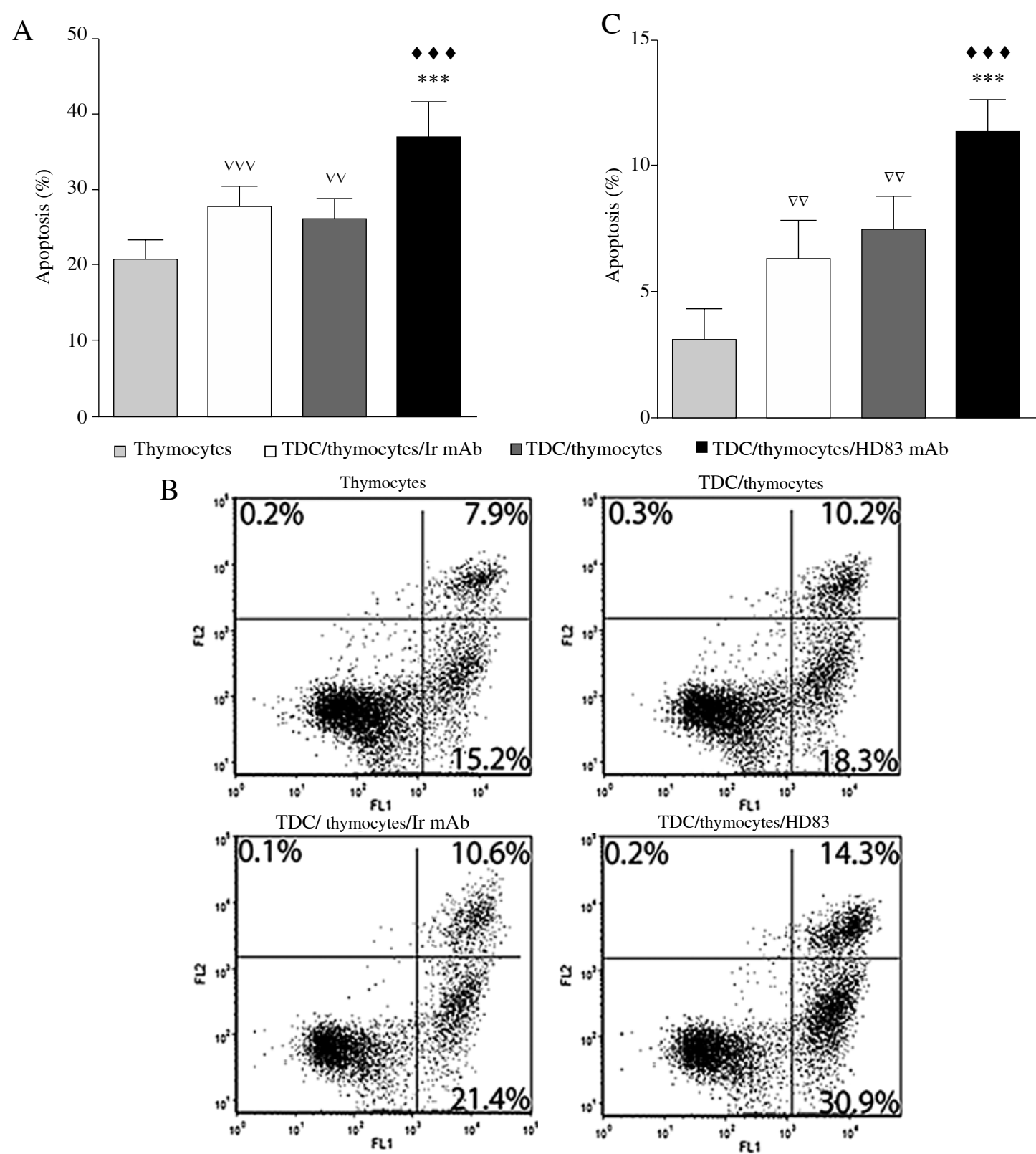

${ }^{* * * *} p<0.001$ compared to Ir mAb, ${ }^{* *} p<0.001$ compared to TDC/thymocytes, ${ }^{*} p<0.01 ;{ }^{* *} p<0.001$ compared to thymocytes alone

Fig. 4. Effect of HD83 mAb on thymocyte apoptosis. Apoptosis of total thymocytes in culture with TDC after 6 hours of cultivation determined by morphological criteria (A), and flow cytometry staining with AnnexinV-FITC/PI (B). The percentage of apoptotic thymocytes within rosettes formed after $30 \mathrm{~min}$ compared to total thymocytes $(\mathbf{C})$ determined by morphological criteria. The results were given as mean \pm SD (3 different experiments) after 500 thymocytes (A), 5000 thymocytes (B) or 200-500 thymocytes within rosettes (C) were calculated in each experiment

It is also possible that HD83, upon binding to DEC205 on TDC, modulates other adhesion molecules on these cells. Our previous results in the rat system, showed that a number of mAbs, including those to class II MHC molecules and CD45, stimulated the rosette formation between TDC and thymocytes in a LFA-1-dependent and LFA-1-independent pathway, respectively [23].
In conclusion, our results showed that the expression pattern of DEC-205 in the rat thymus is very similar to that in the mouse. In addition, we have addressed for the first time a possible role of rat DEC-205 in thymocyte apoptosis through increased TDC-thymocyte interactions. Although preliminary, these results suggest the involvement of DEC205 in the processes of thymocyte selection in the thymus. 


\section{Acknowledgments}

We are grateful to Dr. Chae Gyu Park (Yonsei University College of Medicine, Korea) for a generous gift of HD83 monoclonal antibody.

The authors declare no conflict of interest.

This study is supported by the grants (ON175102) of the Ministry of Education, Science and Technological Development of $R$. Serbia and the project of the Ministry of Defense of R. Serbia (MFVMA/4/13-15).

\section{References}

1. Ladi E, Yin X, Chtanova T, Robey EA (2006): Thymic microenvironments for $\mathrm{T}$ cell differentiation and selection. Nat Immunol 7: 338-343.

2. Starr TK, Jameson SC, Hogquist KA (2003): Positive and negative selection of T cells. Ann Rev Immunol 21: 139-176.

3. Takahama Y (2006): Journey through the thymus: stromal guides for T-cell development and selection. Nat Rev Immunol 6: 127-135.

4. Inaba K, Swiggard WJ, Inaba M, et al. (1995): Tissue distribution of the DEC-205 protein that is detected by the monoclonal antibody NLDC-145. Cell Immunol 163: 148-156.

5. Jiang W, Swiggard WJ, Heufler C, et al. (1995): The receptor DEC-205 expressed by dendritic cells and thymic epithelial cells is involved in antigen processing. Nature 375: 151-155.

6. Kato M, McDonald KJ, Khan S, et al. (2006): Expression of human DEC-205 (CD205) multilectin receptor on leukocytes. Int Immunol 18: 857-869.

7. East L, Isacke CM (2002): The mannose receptor family. Biochim Biophys Acta 1572: 364-386.

8. Park CG, Rodriguez A, Ueta H, et al. (2012): Generation of anti-human DEC205/CD205 monoclonal antibodies that recognize epitopes conserved in different mammals. J Immunol Methods 377: 15-22.

9. Vasilijić S, Colić M, Vucević D (2003): Granulocyte-macrophage colony stimulating factor is an anti-apoptotic cytokine for thymic dendritic cells and a significant modulator of their accessory function. Immunol Lett 86: 99-112.

10. Colić M, Gasić S, Vucević D, et al. (2000): Modulatory effect of 7-thia-8-oxoguanosine on proliferation of rat thymocytes in vitro stimulated with concanavalin A. Int J Immunopharmacol 22: 203-212.

11. Kraal G, Breel M, Janse M, Bruin G (1986): Langerhans' cells, veiled cells, and interdigitating cells in the mouse recognized by a monoclonal antibody. J Exp Med 163: 981-997.

12. Witmer-Pack MD, Swiggard WJ, Mirza A, et al. (1995): Tissue distribution of the DEC-205 protein that is detected by the monoclonal antibody NLDC-145. Cell Immunol 163: 157-162.

13. Swiggard WJ, Mirza A, Nussenzweig MC, Steinman RM (1995): DEC-205, a 205-kDa protein abundant on mouse dendritic cells and thymic epithelium that is detected by the monoclonal antibody NLDC-145: Purification, characterization, and N-terminal amino acid sequence. Cell Immunol 165: 302-311.

14. Iyoda T, Shimoyama S, Liu K, et al. (2002): The CD8+ dendritic cell subset selectively endocytoses dying cells in culture an in vivo. J Exp Med 195: 1289-1302.
15. Kampinga J, Berges S, Boyd RL, et al. (1989): Thymic epithelial antibodies: immunohistological analysis and introduction of nomenclature. Thymus 13: 65-173.

16. Mahnke K, Guo M, Lee S, et al. (2000): The dendritic cell receptor for endocytosis, DEC-205, can recycle and enhance antigen presentation via major histocompatibility complex class II-positive lysosomal compartments. J Cell Biol 151: 673-683.

17. Klein L, Hinterberger M, Wirnsberger G, Kyewski B (2009): Antigen presentation in the thymus for positive selection and central tolerance induction. Nat Rev 9: 833-844.

18. Small M, Kraal G (2003): In vitro evidence for participation of DEC-205 expressed by thymic cortical epithelial cells in clearance of apoptotic thymocytes. Int Immunol 15: 197-203.

19. Lahoud MH, Ahmet F, Zhang JG, et al. (2012): DEC-205 is a cell surface receptor for $\mathrm{CpG}$ oligonucleotides. Proc Natl Acad Sci U S A 109: 16270-16275.

20. Shrimpton RE, Butler M, Morel AS, et al. (2009): CD205 (DEC-205): A recognition receptor for apoptotic and necrotic self. Mol Immunol 46: 1229-1239.

21. Tadic V, Colic M, Miyasaka M, Ilic V. 7-LFA-1/ICAM-1 adhesion pathway is involved in both apoptosis and proliferation of thymocytes induced by thymic dendritic. In: Immunoregulation in health and disease, experimental and clinical aspects. Lukic M, Colic M, Mostarica-Stojkovic M, Cuperlovic K (eds.). Academic Press, London 1997; 77-89.

22. Aiello S, Noris M, Piccinini G, et al. (2000): Thymic dendritic cells express inucible nitric oxide synthase and generate nitric oxide in response to self- and alloantigens. J Immunol 164: 4649-4658.

23. Colić M, Ilić V, Pavlović MD, et al. (1996): Mechanisms involved in the binding of thymocytes to rat thymic dendritc cells. Dev Immunol 5: 37-51. 